\title{
COMMUNICATION
}

\section{Lésions tissulaires dues à l'hydroxyde de calcium : revue de la littérature et suivi d'un cas}

\author{
Lan $\mathbf{R}^{1}$, Hadj-Said $\mathbf{M}^{1}$, Ordioni $\mathbf{U}^{1}$, Catherine $\mathrm{JH}^{1,2}$ \\ 1 - Service de chirurgie orale, CHU Timone, Marseille \\ 2 - UMR 7268 ADES Aix-Marseille Université/EFS/CNRS, Marseille \\ anromain@live.fr
}

Mots Clés : hydroxyde de calcium, nécrose tissulaire, paresthésie

L'hydroxyde de calcium est largement utilisé en endodontie comme médicament intracanalaire pour la désinfection des canaux, comme facteur d'ostéogénèse en cas de lésions périapicales ou permettant de favoriser l'apexification et l'apexogénèse des racines de dents immatures. La forme la plus répandue et la plus efficace est l'hydroxyde de calcium en suspension aqueuse, matériel homogène et peu cytotoxique altérant peu le cycle de vie cellulaire. Ce produit demeure actif durant une longue période et doit son effet antibactérien à son pH élevé. Cependant, une insertion iatrogène de ce biomatériau par défaut d'insertion ou méconnaissance anatomique audelà ou en dehors du canal radiculaire peut entraîner des nécroses des muqueuses ou de la peau, des kystes des maxillaires ou encore des paresthésies du nerf alvéolaire inférieur, sous orbitaire ou mentonnier.

Une patiente de 79 ans a été adressée pour une tuméfaction de la lèvre inférieure. L'examen clinique révélait une tuméfaction locale de la lèvre inférieure avec paresthésie partielle et un œdème intra buccal avec fistule et érythème en regard de la canine mandibulaire droite. Aucune douleur n'était ressentie par la patiente. L'examen tomodensitométrique objectivait une communication endo-parodontale au niveau de la résorption interne de la canine ayant entraîné une fusée importante d'hydroxyde de calcium en sous périosté $(20 \times 10 \mathrm{~mm})$ inséré une semaine auparavant lors d'un traitement endodontique par instrument rotatif mécanique. Une intervention chirurgicale de retrait de l'ensemble du matériau a alors été réalisé avec nettoyage antiseptique soigneux et conservation de la dent causale. Les suites opératoires ont été simples avec nette diminution de la tuméfaction, l'analyse histologique a révélé la présence d'un matériau exogène et une absence totale de paresthésie 2 mois après l'intervention chirurgicale a été constatée.

De nombreux cas de lésions tissulaires due à l'hydroxyde de calcium ont été rapportés dans la littérature depuis plus de 20 ans pouvant entraîner de très graves et longues séquelles comme la paralysie faciale, d'importantes nécroses tissulaires à distance du site d'insertion ou encore la perte de la vue. Selon le degré d'atteinte, certains cas pourront nécessiter le recours à la chirurgie afin d'assurer une décompression tissulaire, de faciliter le drainage mais aussi de créer de nouvelles conditions environnementales propices à la guérison. De plus, ce type d'intervention a pour avantage d'apporter une irrigation directe au niveau du site endommagé et d'assurer l'élimination des débris tissulaires nécrotiques. II semble ainsi essentiel de savoir gérer les conséquences d'un tel événement de manière à pouvoir mettre en place une prise en charge 